\title{
Bemühen um Japan - das Vermächtnis Hans Boeschs
}

Hans Boesch: Japan. Kümmerly \& Frey, Bern 1978, 257 S., 29 Abb., 42 Bildtafeln. Fr. 48.--.

Es ist in den letzten Jahren üblich geworden, geisteswissenschaftlich-hermeneutische Verfahren und Arbeitsweisen im Rahmen der geographischen Arbeit strikt abzulehnen und abzuwerten. Daran ist gewiss richtig, dass sich eine geographische Länderkunde nicht auf Hermeneutik allein gründen kann und sich vor allem nicht auf sie zurückziehen darf, wenn es in der Arbeit schwierig und unbequem wird. Beim Kulturvergleich wird man jedoch nur zum Schaden der Länderkunde auf geistiges Bemühen um Verstehen und Einfühlen ganz verzichten können. Vor allem bei der sozialgeographischen Analyse fremder Gese11schaften und ihrer Entwicklungsprägungen werden qualitative Kriterien so bedeutsam, dass andere, voll objektivierbare Verfahren zu ihrer Erfassung meist nicht ausreichen.

Als subjektive Voraussetzung zur Erkenntnis in alten, differenzierten Hochkulturen wird auch auf menschliche Verhaltensweisen wie Intuition und Takt kaum zu verzichten sein. Wer einmal erlebt hat, wie sich renomierte und bedeutende Sozialwissenschaftler in einem ihnen fremden Kulturkreis über längere Zeit hinweg wie Elefanten in einem Porzellanladen bewegten, ohne viele der durch sie bedingten Scherben überhaupt wahrzunehmen, der wird diese Lektion sein Leben lang nicht vergessen. Doch auch an der eigenen Person wird man bei kritischer Selbstbeobachtung herausfinden, dass gewisse Einstellungen und Verhaltensweisen geradezu Voraussetzung dafür sind, dass man in fremden Kulturkreisen Zugang zu Erkenntnissen findet.

Hans Boesch hat in seiner Landeskunde von Japan über derartige Fragen und Zusammenhänge nicht theoretisiert. Für ihn ist es selbstverständlich, dass ein Land wie Japan mit seiner Gesellschaft, Geschichte und Kultur lange, intensive Bemühungen um 'Verstehen" erfordert, ohne dass der Erfolg immer sicher ist. Dabei wird der Leser immer wieder in die Reflektion des Autors miteinbezogen; es wird einsichtig - und das macht das Buch so sympatisch und fesselnd -, dass ein Versuch, die Gesamtzusammenhänge japanischen Lebens darzustellen, in mancher Hinsicht unvollkommen bleiben muss. 'Manches musste unausgesprochen bleiben, entweder, weil uns das Gespräch zu weit weg vom eigentlichen Thema geführt hätte oder weil einfach viele Zusammenhänge undurchsichtig sind" (S.253).

Ungewöhnlich und interessant ist der Aufbau des Buches. Entsprechend der Zielsetzung, dem Verständnis des westlichen Lesers die besondere Situation dieses Landes näherzubringen, stehen die Fragen des gesellschaftlichen und wirtschaftlichen Umbruchs im Vordergrund. So beginnt die Darstellung nach einem kurzen, sehr überlegt und wesentlich gerafften geschichtlichen Ueberblick ("Begegnung mit Japan", nur 6 Seiten) und einem kurzen Abschnitt "Gedanken zu einer Länderkunde von Japan" mit dem Thema Verstädterung. Es folgen, entsprechend ihrer Bedeutung in der gegenwärtigen Situation, Industrien und Dienstleistungen, Urproduktion sowie Aspekte und Möglichkeiten der Regionalisierung. Nach einem Kapitel über gesellschaftliche Strukturen und Prozesse: (Image, Hausgemeinschaft, Erziehung und Berufsleben) schliesst das Buch mit Gesichtspunkten der Lage, Einordnung und Aussenkontakte: "Japan in der Welt von heute". Die Physische Geographie wurde jeweils dort berücksichtigt, "wo sie zum Verständnis der behandelten Fragen wesentlich beitragen konnte". (S.27)

Auch innerhalb der Kapitel ist vieles unkonventionell konzipiert und gegliedert. Besonders gut gelungen und geographisch verknüpft scheint mir die Behandlung von Fischerei, Land- und Forstwirtschaft unter den drei Raumkategorien: "Küs乞e und Meer", "Die Ebenen und ihre Umrandung"' und "Bergland". Das ist ein landeskundlich-integrativer Ansatz, der trägt und tiefere Einsichten erlaubt, als eine konventionelle Abfolge allgemeiner Gesichtspunkte. Auch die statistische $\mathrm{Zu}-$ ordnung gelingt hier fast bruchlos.

Vorbildlich ist die Ausstattung des Buches mit Karten und farbigen Bildbeigaben, meist sehr aussagekräftigen Luftaufnahmen vom Geographical Survey Institute in Tokyo. Die überlegt konzipierten Schwarz-Weiss-Kärtchen und Tabellen zeugen vom Blick für das Wesentliche. Besonders originell sind die graphischen Darstellungen des "Streikfahrplans" im Verkehrswesen während der Frühjahrsoffensive der Gewerkschaften(Fig.28), des Schnellbahnsystems Shinkansen (Fig.8), und des japanischen Meeres-Wirtschaftsraumes als Souveränitätsbereich (Fig.25).

Prof. Dr. Peter Schöller, Geograph. Institut, Ruhr-Universität Bochum, Universitätsstr. 150, Postfach 1021 48, D - 4630 Bochum 1 
Im Kapite1:"Land, Landschaft, Regionen" wird der argumentative Charakter des Werkes besonders deutlich. Auch wenn mir die Sachaussagen dabei durchaus nicht immer eindeutig, treffend und überzeugend scheinen, die Einstellung aufs "Japanische" gelingt eindrucksvoll. Die stete Auseinandersetzung mit Begriffen, ihrer Uebersetzbarkeit und Problematik, die Unterschiede des Denkens in West und Ost schaffen nicht nur Transparenz, sondern führen oft zum Ansatz eines Kulturvergleichs. So ist es durchaus bezeichnend, dass sich an eine Behandlung der Regionalisierung zwanglos Ausblicke auf Besonderheiten der Entwicklung der japanischen Geographie als Wissenschaft ergeben.

Auf die Frage des sozialen Zusammenlebens wird mit Recht immer wieder eingegangen. Hier gelingen Hans Boesch gut formulierte Einsichten:"Für den Japaner spielt immer und jederzeit eine entscheidende Rolle, dass er sich über seine eigene Stellung innerhalb der Gesellschaft im klaren ist. Auch die Religion lehrt nicht die Gleichheit aller Menschen; der Buddhismus akzeptiert, dass die Menschen ungleich sind, und dass sie sich mit ihrer Stellung abfinden und darin glücklich sein müssen. Eine sogenannte niedrige Stellung in der Gesellschaft hat deshalb auch nichts Entwürdigendes; dagegen kann man sich in jeder Stellung sowohl richtig wie auch falsch benehmen, glücklich oder unglücklich sein, wichtig ist, dass man seine eigene Position kennt und, wenn man mit anderen zusammentrifft, sich über die gegenseitige Beziehung im klaren ist. In dieses Netz von Beziehungen wird der junge Japa$n \in \mathrm{r}$ schon in seinen frühesten Jahren einbezogen und weiss sich entsprechend zu verhalten. Für einen fremden Besucher Japans ist freilich das Erkennen dieser Strukturen etwas vom Schwierigsten, und darum benimmt man sich auch so häufig unhöflich und unmöglich". (S.210)

"Die Zugehörigkeit zu einer Gruppe spielt für den Japaner eine wichtige Rolle; dabei gibt es für ihn nicht nur eine, sondern in der Regel eine gewisse Zahl von Gruppen, denen er sich verbunden fühlt. Die Haus- oder Familiengemeinschaft ist die erste, in die er hineinwächst, doch wird sie später durch andere ergänzt und teilweise abgelöst. Ausserhalb der Gruppe füh1t sich ein Japaner 'out of place' und verliert leicht die Orientierung. Oft benimnt er sich dann gehenmt, weil er glaubt, dass er sich ungeschickt und falsch verhält. Darum spielt auch der andere Ausdruck, den wir verwendeten, Geborgenheit, eine besondere Rolle. Der Japaner ist glücklich in der Geborgenheit seiner Gruppe, wo er - ganz im Gegensatz zum Eindruck, den er etwa im Ausland erweckt - aufleben und fröhlich sein kann'. (S.210f.)
Besonders überzeugend wird der Text auch da, wo allgemeine Aussagen mit subjektiven Erlebnissen und Eindrücken illustriert werden: Kontakte mit Grundschulklassen verschiedenen Geschlechts (auf S.212), Vergleich von Autoausflügen 1955 und 1965 (S.95) oder Preisbeobachtungen bei Lebensmitteln (S.119). An anderen Stellen freilich fehlen gerade vertiefte Differenzierungen, wie sie sich aus eigenen Feldforschungen ergeben würden. So ist etwa die Bedeutung der ländlichen und städtischen Traditionsgewerbe nicht gesehen und gewürdigt; die "Textilindustrie" müsste aufgegliedert werden; das Verkehrskapitel ist zu uneinheitlich; die Angaben zur Wohnungsmiete (S.34) sind irreführend; Jutaku-kodan sollte keinesfalls als "öffentliche Baugenossenschaft" übersetzt werden (S.197); auch ist nicht klar, warum die Frage, ob ein 'Megalopolis-Bewusstsein" entsteht, "entscheidend" sein soll (S.56).

Doch das sind Einzelheiten, die gering wiegen bei einem so umfassenden, problemorientierten Gesamt-Konzept. Entscheidend für den Erfolg ist sicher die langjährige Beschäftigung des Autors mit seinem Thema und die häufigen, im Abstand von wenigen Jahren wiederholten Besuche des Landes sowie die Hilfe bedeutender wissenschattlicher Mentoren aus dem Lande selbst. Doch diese Anschauung und Landeskenntnis verbindet sich mit Voraussetzungen in der wissenschaftlichen Persönlichkeit des Autors:-Erfahrung, Weitsicht, Originalität, Urteilsfähigkeit, Problembewusstsein.

So ist sein Buch keine Faktendarbietung geworden, keine sezierende Analyse, keine oberlehrerhafte Verkündigung, sondern eine sehr lesbare, fast erzählende Darstellung, oft bewusst impressiv, mit Gespür für Mentalität und Stimmungswerte, argumentativ und transparent in der Auseinandersetzung mit Befunden und Begriffen und den verschiedenen Traditionen des Denkens in Ost und West, gerade das macht das Buch wichtig, als Schlüssel und Tor zum Verständnis Japans.

Durch das Dickicht der Fakten und verfilzten Zusammenhänge hat Hans Boesch inmer wieder Schneisen geschlagen, Blickachsen geschaffen, Aussichtspunkte erklommen. Dadurch mag manche Einzelheit unscharf werden und andere Beziehungen müssen fehlen; doch umso deutlicher werden die Durchblicke, die Akzente und Leitlinien. Auch erlauben diese Schneisen den Blick zurück auf den Autor selbst. Die herbe, zuweilen spröde und verschlossene, eigenwillige Persönlichkeit Boeschs zeigt hier andere Züge, die wohl nur Vertraute von ihm kennen: Sensibilität, Verletzlichkeit, Bescheidenheit. Es ist ein lauteres, ehrliches Buch, eine Länderkunde, geschrieben mit Geist und init Herz; für uns ist sie nach dem plötzlichen Tode Hans Boeschs ein Vermächtnis, für das wir dankbar sind. 